\title{
The AtGentive project: Attentive Agents for Collaborative Learners
}

\author{
Claudia Roda ${ }^{1,3}$, Thierry Nabeth ${ }^{2,3}$ \\ ${ }^{1}$ American University of Paris, Computer Science Department, \\ 112, rue du Bac, 75007 Paris, France \\ crodalaup. fr \\ ${ }^{2}$ INSEAD Centre for Advanced Learning Technologies, \\ Bvd de Constance, 77305 Fontainebleau Cedex, France \\ thierry. nabeth@insead.edu \\ ${ }^{3}$ AtGentive consortium \\ http: //www.atgentive.com/
}

\begin{abstract}
Attention, which intervenes at many different levels such as the perception of the environment and the allocation of cognitive resources, appears to represent one of the key factors of learning and working performance. This poster presents AtGentive, a project which aims at investigating the use of agent-based ICT systems for supporting the management of the attention of young or adult learners in the context of learning and collaborative environments.
\end{abstract}

\section{Introduction}

Attention appears to represent one of the key factors of learning or working performance. The most effective learners and knowledge workers are often those people capable of filtering and selecting the most relevant information and at allocating their cognitive resources in the most appropriate manner.

This ability to manage efficiently attention can be considered as even more critical in the new learning and working contexts [see for example 1, 2, and 3]. For instance, in an online learning setting, the learners are more on their own; they have less guidance; and cannot situate themselves with others and adjust their behaviour. Even in presence of strong commitment, it is harder to evaluate optimal time allocation, and effectiveness of learning or collaborative processes. In this context, the learners have fewer points of reference to situate themselves, may not receive any direct pressure from a tutor or from their peers, and can more easily procrastinate, or engage into learning activities that are very ineffective. In the "knowledge economy", employees are engaged in a multitude of projects involving a variety of actors from different horizons with which they have to collaborate. They also have to process more information and solicitation than in the past, originating from a variety of sources, and available in different forms (news, email, instant messaging, etc.). Finally, they are more autonomous and more responsible for their lines of actions. As a consequence, the knowledge workers have more risk to be overwhelmed by too much information 
and too many interruptions, and also to manage inefficiently the execution of the many tasks they have to accomplish for their work [1]. These new conditions typically results in a situation of information and cognitive overload for the learners and for the knowledge workers that represent a real challenge that need to be addressed so as to facilitate the setting up and the adoption of new methods of learning and work in the Information Society.

The AtGentive project is born from the idea that such a challenge can be addressed with the help of ICT systems that are aware of this attentional dimension, and that are able to support the individuals or the groups at filtering and selecting the most relevant information for them and helping them allocating their cognitive resources. Practically, the objective of this project is to investigate how to design such attention supporting systems, and in particular to explore how artificial agents, that may be embodied into artificial characters, can be used to provide more active and intelligent support to attention.

The first part of this poster will present the AtGentive project: what are the objectives of this project, what are the principles that will be used to design attention supporting systems for elearning and collaborative working context. The second part of this poster will present how these principles are going to be applied to design and to test "attention support" in two applications: (1) AtGentSchool: an elearning application for child education; (2) AtGentNet: an advanced virtual community platform supporting knowledge exchange of communities of distributed managers. The last part of this poster will conclude, and will indicate the future work.

\section{A project aiming at supporting individual and group attention}

\subsection{An Overview of the AtGentive project}

The AtGentive project explores the links between learning and attention and it includes: (1) the definition of a model of attention for the learner (low-level \& high level, individual \& social); (2) different mechanisms to capture or infer information about the state of attention (both at a low and high level) of the user in agentenhanced collaborative learning platforms; (3) Intervention mechanisms using attentive agents informed about users attentional state, providing guidance, helping users to better manage their attention to achieve their learning objective, reducing information overload, and therefore improving the effectiveness of the learning \& working process.

More specifically, the important foci of the project include:

- A model of attention of the user, and the support of this attention by attention informed systems. A deep understanding of attention (importance, impact, processes to support it, etc.) will be elaborated

- The "sensing" of the environment and in particular the different means for collecting information that can be used to profile the user's attentional state. 
- The design of a set of mechanisms for supporting the management of the attention of users and groups (for instance enhancing the user perception).

- The design of an artificial agent cognitive architecture able to proactively and intelligently support the user attention.

Practically, the technical infrastructures that will be designed in this project will consist in ICT platforms that are enhanced with different components (intelligent or not) providing different level of attentional support such as:

- Components and approaches helping to enhance the perception of the user by filtering or emphasizing the information presented or delivered to the user, and therefore contributing to the reduction of information and cognitive overload. Examples of such approaches include the information design of the spaces facilitating the user perception, the use of visual tags emphasizing the most important items, or the ordering of the information according to the importance of the item (relevance for the user, freshness of the item, popularity of the item).

- Components and agents facilitating the organization of learning and work processes. For instance, this support can be provided for the execution of activities in presence of multitasking, frequent interruptions, and large information sources. Examples of such mechanism include enhanced notification mechanisms and interruption management (e.g. delaying a notification, or delivering it in a way that does not break the concentration of the user), and the support of task resumption (saving and restoring a context for a task that has been interrupted).

- Intelligent agents and mechanisms able to coach the learners or the knowledge worker in reaching higher level of performance in managing their attention. These agents will in particular help the user to acquire attention management skills. Examples of these agents and mechanisms can include the provision of statistics displaying the different activities of the user and the time he/she has allocated to them, but also proactive agent interventions making suggestions to the user (for instance the agent may suggest a new focus, or may encourage the user to adopt a more effective learning or communication practice)

\subsection{A First Sketch of the AtGentive internal architecture}

The agents, which may appear embedded in a artificial characters, are able to profile the learners' attentional state (short or long term) by observing their actions. On the basis of this profile, Atgentive agents can assess, analyze, and reason about user's attention, and provide assistance (for instance automating some tasks) and proactive coaching (assessment, guidance, stimulation, etc.).

Fig 1. represents a first sketch of the AtGentive architecture that will be further elaborated and then implemented during the project. 


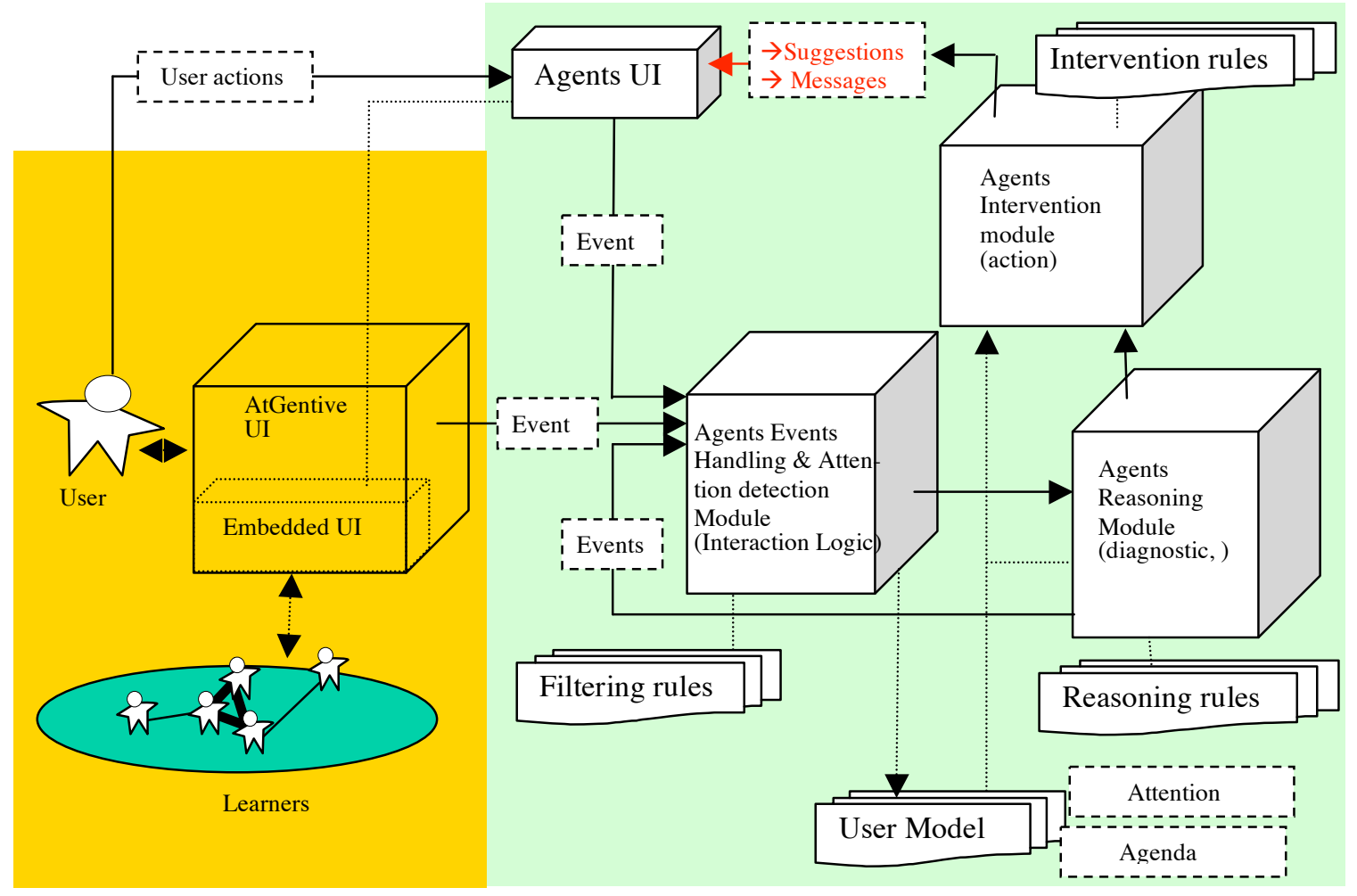

Fig. 1. A first sketch of the AtGentive internal architecture

The main components of this architecture are:

- The attention detection module which monitors and profiles the learner's attentional state.

- The attention reasoning module. This module represents the main intelligent reasoning part. Its function is to (1) Assess the situation; (2) Identify and build a selection of possible assistance and coaching interventions.

- The Intervention module. The function of this component is to select and execute the interventions that are the more appropriate (having the maximum impact on the user, and do not unnecessary distract the user).

- The interface. The user interface will rely on well designed interaction portals and artificial characters (artificial agents may intervene as embodied agents) that are attention "friendly".

\section{3: AtGentSchool and AtGentNet: Two applications applying the AtGentive principles}

Two different "attentive" applications are used to validate the principles elaborated in the AtGentive project: (1) AtGentSchool: an active elearning platform for child education; (2) AtGentNet: an advanced virtual community platform. 


\subsection{AtGentSchool: an "Attentive" active elearning platform for child education}

AtGentSchool is an e-learning application for child education that will be built upon on the existing Ontdeknet system [4]. Ontdeknet is a learning environment founded on learning arrangement principles [5], an educational approach based on constructivism, situatedness, and active engagement into collaborative learning activities.

The incorporation of the support of attention is expected to improve the effectiveness of these systems by increasing the level of involvement of the learners, and leading to a more effective learning process. This objective will be accomplished by having the opportunity to measure and to assess the individual activity and attention patterns, during the learning arrangement, and later to allow adaptive adjustments to the learning objects and their interconnections.

A number of advantages are expected for the AtGentSchool users:

- The behaviour of the existing embodied agent (whose role is to help children through the learning process) will appear more natural to the user, which will enhance acceptance and prevent (the Microsoft dog) irritation.

- The agent behaviour will become adaptive, resulting in more efficient guidance

- Adaptation to the user is expected to enhance motivation

- More effective guidance and higher motivation are expected to lead to more effective learning experiences

\subsection{AtGentNet: an "Attentive" advanced virtual community platform for supporting knowledge exchange}

AtGentNet will consist in the design of an advanced virtual community platform supporting knowledge exchange of communities of distributed managers, relying on a model structuring of the communication using spaces currently implemented in the ICDT platform [6].

The incorporation of the support of attention in the ICDT platform will result in a set of mechanisms helping to enhance communication and knowledge exchange inside the platform.

Examples of mechanisms include:

- Advanced communication filtering and management mechanisms helping to reduce the knowledge and information overload associated with interaction with the other members of the community.

- Social awareness and translucence mechanisms displaying the activity happening in the community, helping to orient the attention of the users.

- Advanced monitoring capabilities (capturing the actions of the users, and basic filtering about these behaviours conducting to the generation of events that can be exploited by human or artificial agents)

- Artificial agents having some capacities to observe and to reason about the environment and about the users and to intervene proactively.

- "Attentional" expertise, allowing the agents to support managers in managing their own attentional state, both at the low level, and at the higher cognitive level.

- Artificial character interface, displaying agents as an anthropomorphic characters. 


\section{Conclusion and future work}

The project has already started, with the involvement of the end users, to draft a series of scenarios. Such scenarios explore different ways to provide support for attention in an ICT platform. A few of the mechanisms have already been incorporated into first prototypes.

The AtGentive project is still at a too early stage to draw any definitive conclusions. However we have already observed that the concept of attention, by providing a new angle of analysis taking into account deeply human cognition, appears to be very useful to guide the design of mechanisms addressing the real issues that people are facing today (such as information overload, and the complexity of user-computer interaction). In some cases the concept of attention has provided us with a new angle of analysis of existing mechanisms that were not initially intended for supporting attention.

The second phase of this project, and its validation with the different pilots (one in schools, the second in a learning network), will help us to better understand which are the mechanisms supporting attention that are the most useful, and in particular will help us to assess the effectiveness of the new mechanisms (such as agent-based support) that will be elaborated for this projects.

Acknowledgments. The work described in this paper was partially sponsored by the EC under the FP6 framework project Atgentive IST-4-027529-STP. We would like to acknowledge the contribution of all project partners including: Laurent Ach, Albert A. Angehrn, Jaroslav Cech, Eugeni Gentchev, David Kingma, Pradeep Kumar Mittal, May Liem, Ivana Mala, Inge Molenaar, Koen Molenaar, Benoit Morel, Thierry Nabeth, Barbora Parrakova, Paul Rudman, Hari Siirtola, Toni Vanhala, Maurice Vereecken, Deng Ye, Mary Zajicek.

\section{References}

1. Davenport, Thomas and Beck John (2001); The Attention Economy; Harvard Business School Press, 2001

2. Roda, C., \& Nabeth, T. (2005). The role of attention in the design of learning management systems. Proceedings IADIS International Conference CELDA (Cognition and Exploratory Learning in Digital Age), Lisbon, Portugal, 148 - 155.

3. Roda, C., \& Thomas, J. (2006). Attention aware systems: Theories, applications, and research agenda. Computers in Human Behavior, 22(4), 557-587.

4. Ontdeknet: http://www.ontdeknet.nl/

5. Simons, P.R.J., Van der Linden, J.L., \& Duffy, T. (Eds.) (2000). New learning. Dordrecht: Kluwer.

6. Albert A. Angehrn (2004); Designing Effective Virtual Communities Environments: The ICDT Platform; INSEAD CALT Report 10-2004 